\title{
A New Command Shaper Design for Residual Vibration Reduction in Flexible Systems Using Artificial Mode Constraints
}

\author{
Seong-Wook Hong, Member, IACSIT, Yong-Kyu Seo, and William Singhose
}

\begin{abstract}
Positioning systems often exhibit vibration resulting from changes in speed and direction. Command shaping efficiently reduces residual vibration by generating appropriately shaped reference commands for positioning systems. However, the presence of command shapers increases the rise time of the systems. This paper proposes a new command-shaper design method for a single-mode system. In addition to suppressing the dominant single mode, this method also constrains vibration at an artificial high mode. This approach can shorten the command shaper duration, or the rise time of the system. The artificial mode frequencies are design variables used to adjust the rise time of the system. The proposed design scheme is illustrated with a simulation example.
\end{abstract}

Index Terms-Artificial mode constraint, command shaping, natural frequency, residual vibration

\section{INTRODUCTION}

Positioning systems such as XY stages, industrial cranes, robotic manipulators, and so on, experience residual vibration when they increase their operational speed or change their moving direction. Many researchers have developed control methods that can reduce such residual vibration in positioning systems. Command shaping has been developed to reduce residual vibration in flexible dynamic systems without the need for feedback control [1]-[6]. This method has distinguishable advantages in implementation: simplicity and low cost [4]. However, when input shaping is implemented, the rise time of the system increases [4], [5]. The rise time increase may be critical in some applications, such as high-speed stages.

Hong, et.al [5] previously introduced a virtual mode concept for command shaper design to adjust the rise time of the undamped vibration system. Previous analysis of robust command shapers has shown that they can be interpreted as cancelling the vibratory mode, and also cancelling another virtual mode at the same frequency [7]. This paper proposes the command-shaper design method using artificial mode constraints for damped single mode systems. The proposed

Manuscript received August 17, 2012; revised October 15, 2012. This work was financially supported by the Research Fund of Kumoh National Institute of Technology.

S. W. Hong is with the Department of Mechatronics, Kumoh National Institute of Technology, Gumi, Gyeongbuk 730-701, Korea (e-mail: swhong@ kumoh.ac.kr).

Y. K. Seo is with the Top Engineering Co., Ltd., Paju, Gyeonggi 413-813, Korea (e-mail: ykseo@topengnet.com).

W. Singhose is with the School of Mechanical Engineering, Georgia Institute of Technology, Atlanta, Georgia 30332, USA (e-mail: singhose@gatech.edu). method is to design a three-impulse command shaper as if the single mode system has two modes. Simulations show that the proposed command shaper design method is very useful for reducing residual vibration and adjusting the rise time of damped single mode systems.

\section{Command Shaper Design Using Artificial Mode CONSTRAINTS}

The basic idea of the method proposed here comes from the multi-mode command shaper design method that was described in [6] by the authors. The proposed method uses a three-impulse command shaper designed to suppress two modes. However, it is used for a single mode system, as if the single mode system has two modes. The method is also related to the work by Singh and Heppler [8], wherein they used 3 impulses to cancel two real vibration modes. Fig. 1 shows a typical three-impulse command shaper. The command shaper for two-mode systems that has three impulses can be written in the Laplace domain as [6].

$$
I(s)=A_{1}+A_{2} e^{-t_{2} s}+A_{3} e^{-t_{3} s}
$$

where $A_{k}$ and $t_{k}, k=1,2,3$, represents the $k^{\text {th }}$ impulse amplitude and its time. The first impulse is assumed to start at time zero, i.e., $t_{0}=0$, without loss of generality.

Consider the following two eigenvalues:

$$
s_{s}=-2 \pi \zeta_{s} f_{s}+j 2 \pi f_{s}^{d}, s_{a}=-2 \pi \zeta_{a} f_{a}+j 2 \pi f_{a}^{d}
$$

where $s_{s}$ and $s_{a}$ are the system eigenvalue to be suppressed and the artificial eigenvalue, respectively. $f_{s}$ and $f_{a}$ are the natural frequency of system and the artificial undamped-mode frequency, respectively, and $\zeta_{s}$ and $\zeta_{a}$ are the associated damping ratios. $f_{s}^{d}$ and $f_{a}^{d}$ are the damped natural frequency of system and the artificial damped-mode frequency, which are defined by $f_{s}^{d}=f_{s} \sqrt{1-\zeta_{s}^{2}}$ and $f_{a}^{d}=f_{a} \sqrt{1-\zeta_{a}^{2}}$.

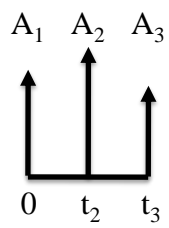

Fig. 1. Typical three-impulse command shaper 
Here, we will introduce a command shaper that can manipulate the input command, thereby lowering excitation. The principle can be explained by pole-zero cancellation [4]-[7]. If all the flexible poles of the system correspond to the zeros of the command shaper, then any reference command applied to the system will not induce residual vibration [2]. Thus, a command shaper that forces the vibration of the two modes to zero should satisfy the following equations:

$$
I\left(s_{s}\right)=0, I\left(s_{a}\right)=0
$$

Equation (3) consists of two simultaneous complex-valued nonlinear equations, each of which provides two real-valued equations. However, since the number of unknowns to be determined is 5 , one more equation is needed to yield meaningful solutions. An additional equation may be provided from a constraint on the sum of impulse amplitudes as follows:

$$
I(0)=A_{1}+A_{2}+A_{3}=1
$$

Equations. (3) and (4) can be rewritten, with the parameters of three impulses:

$$
\left[\begin{array}{ccc}
1 & 1 & 1 \\
1 & e^{-t_{2} s_{1}} & e^{-t_{3} s_{1}} \\
1 & e^{-t_{2} s_{2}} & e^{-t_{3} s_{2}}
\end{array}\right]\left\{\begin{array}{l}
A_{1} \\
A_{2} \\
A_{3}
\end{array}\right\}=\left\{\begin{array}{l}
1 \\
0 \\
0
\end{array}\right\}
$$

The nontrivial solution condition for (5) leads to

$$
\begin{aligned}
& e^{2 \pi\left(\zeta_{s} f_{s} t_{2}+\zeta_{a} f_{a} t_{3}\right)} \sin \left(2 \pi f_{s}^{d} t_{2}\right) \sin \left(2 \pi f_{a}^{d} t_{3}\right) \\
& \quad-e^{2 \pi\left(\zeta_{a} f_{a} t_{2}+\zeta_{s} f_{s} t_{3}\right)} \sin \left(2 \pi f_{a}^{d} t_{2}\right) \sin \left(2 \pi f_{s}^{d} t_{3}\right)=0
\end{aligned}
$$

Set the artificial undamped-mode frequency and damping ratio to satisfy the following relation:

$$
2 \pi \zeta_{a} f_{a}=2 \pi \zeta_{s} f_{s}
$$

Then, (6) is simplified as

$\sin \left(2 \pi f_{s}^{d} t_{2}\right) \sin \left(2 \pi f_{a}^{d} t_{3}\right)-\sin \left(2 \pi f_{a}^{d} t_{2}\right) \sin \left(2 \pi f_{s}^{d} t_{3}\right)=0$

It is convenient to equally space the impulses, such that $t_{3}=2 t_{2}$. Then,

$$
\cos \left(2 \pi f_{s}^{d} t_{2}\right)=\cos \left(2 \pi f_{a}^{d} t_{2}\right)
$$

The solutions for (9) can be written as

$$
t_{2}=\frac{m}{f_{a}^{d} \pm f_{s}^{d}}, \quad m=1,2, \ldots
$$

Thus, the minimum shaper duration can be obtained by

$$
t_{3}=\frac{2}{f_{a}^{d}+f_{s}^{d}}
$$

The associated impulse amplitudes are:

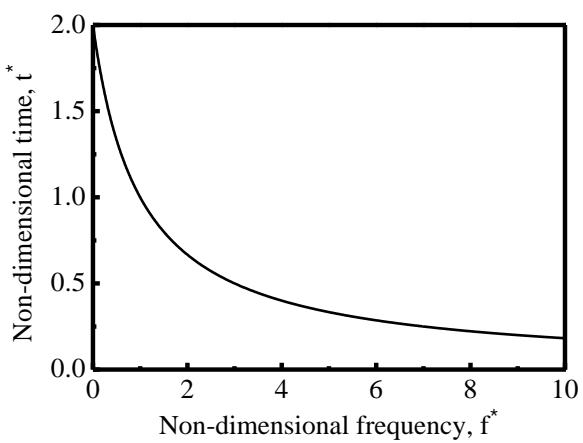

Fig. 2. Command shaper duration vs. artificial mode frequency

$A_{1}=\frac{P^{2}}{1-2 P Q+P^{2}}, A_{2}=\frac{-2 P Q}{1-2 P Q+P^{2}}, A_{3}=\frac{1}{1-2 P Q+P^{2}}$

where $P=\exp \left(\frac{2 \pi \zeta_{s}}{\sqrt{1-\zeta_{s}^{2}}} \frac{f_{s}^{d}}{f_{s}^{d}+f_{a}^{d}}\right), Q=\cos \left(\frac{2 \pi f_{s}^{d}}{f_{s}^{d}+f_{a}^{d}}\right)$

The non-dimensional artificial frequency and shaper duration time, $f^{*}, t^{*}$, are defined as follows:

$$
f^{*}=\frac{f_{a}^{d}}{f_{s}^{d}}, t^{*}=\frac{t_{3}}{T_{d}}=t_{3} f_{s}^{d}
$$

where $T_{d}$ is the fundamental period of vibration. Fig. 2 shows the relation between the non-dimensional artificial frequency and the non-dimensional shaper duration. The figure demonstrates that the non-dimensional artificial frequency has a one-to-one correspondence with the non-dimensional shaper duration. Thus, the artificial mode frequency, $f_{a}^{d}$, can be a design parameter to adjust the shaper duration, or the rise time of the system. The proposed shaper duration becomes shorter as the artificial mode frequency increases. The non-dimensional shaper duration is 0.5 at the nondimensional artificial frequency of 3 . This is identical to a single-mode ZV (Zero Vibration) shaper [4].

Fig. 3 shows the impulse magnitudes of the shaper as a function of the non-dimensional artificial frequency. The impulses, $A_{1}$ and $A_{3}$, are always positive but the sign of the middle impulse, $A_{2}$, changes with frequency. If the non-dimensional artificial frequency approaches 5 , then the impulse magnitude becomes greater than 1 . This is a very important point for command shaper design because the existence of impulse magnitudes greater than 1 can cause saturation to the actuators [9]. However, the shaper duration may be shortened further unless actuator saturation occurs. The damping increases the amplitude of $A_{1}$, but decreases that of $A_{3}$. On the other hand, $A_{2}$ is nearly independent of damping. 

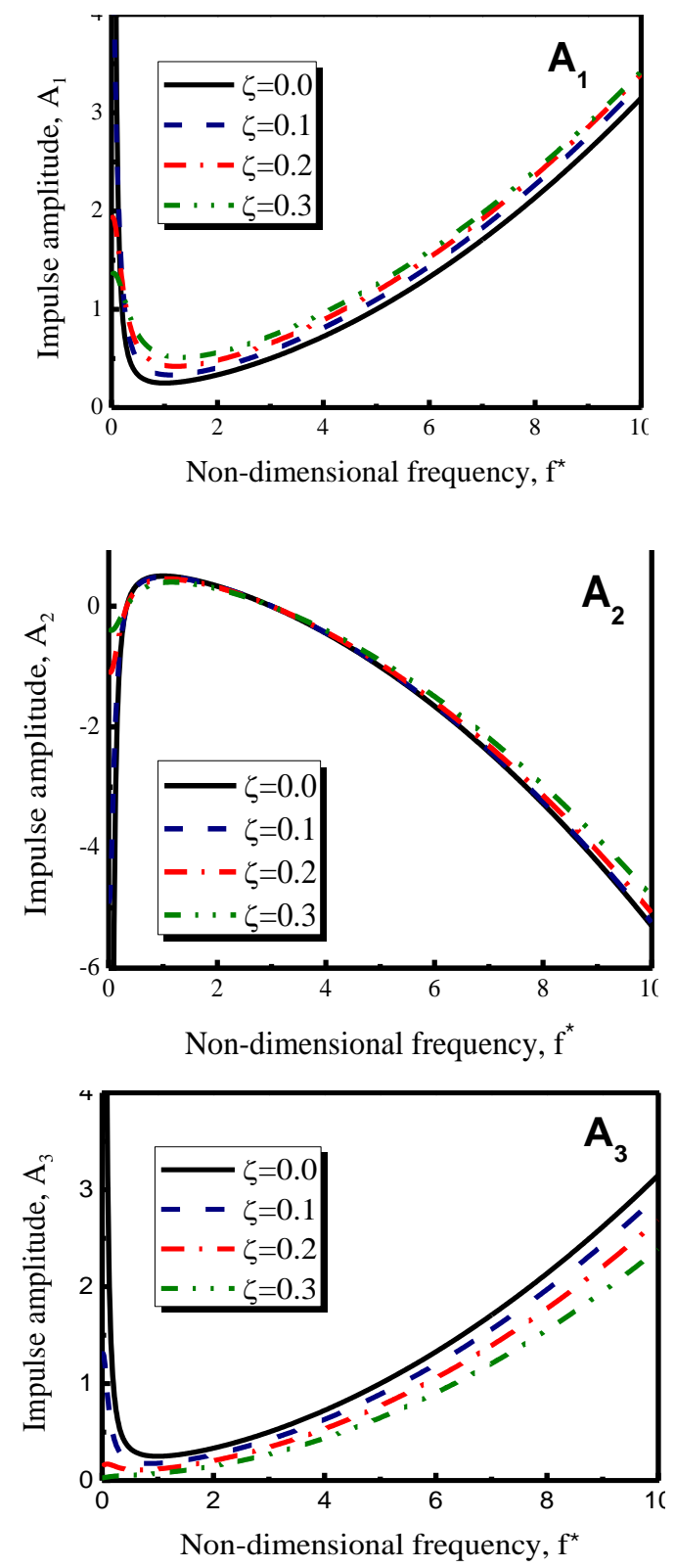

Fig. 3. Impulse amplitudes as a function of non-dimensional artificial frequency

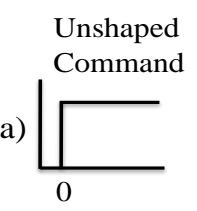

(b)

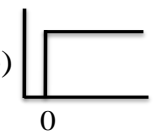

(c)

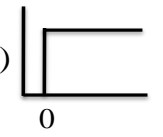

(d)

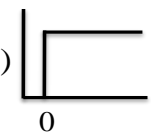

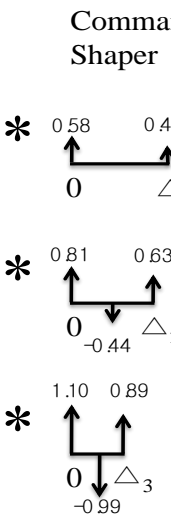

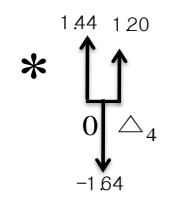

Shaped Command
Fig. 4. Command generation with the proposed shapers (damped natural frequency: $1 \mathrm{~Hz}$, damping ratio: 0.1 )

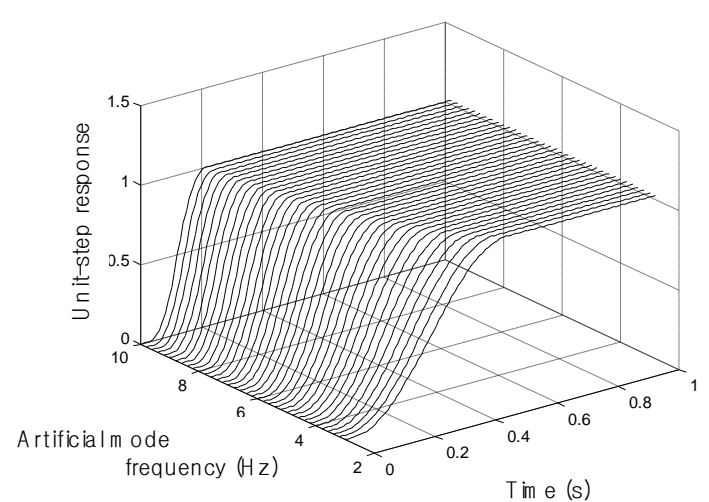

Fig. 5. Shaped unit-step responses with the artificial mode frequency varied (damped natural frequency: $1 \mathrm{~Hz}$, damping ratio: 0.1 )

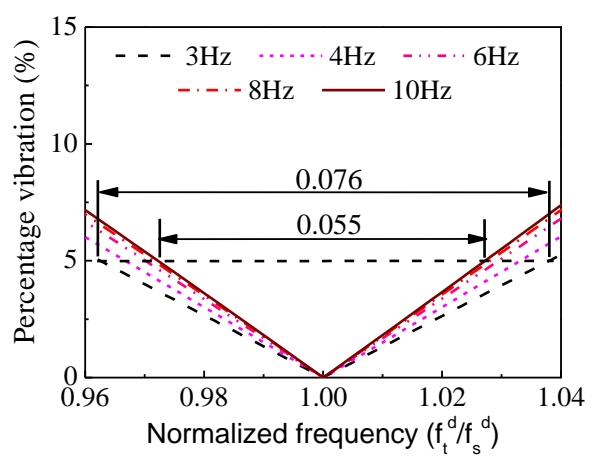

Fig. 6. Sensitivity curves for the proposed command shapers with various artificial frequencies

\section{ILlustration OF THE PROPOSED COMMAND SHAPER}

To illustrate the proposed command shaper design method, Fig. 4 shows four different cases with the proposed method for a damped single mode system of which the damped natural frequency and damping ratio are $1 \mathrm{~Hz}$ and 0.1 .

Fig. 4(a) shows the step command shaped by a command shaper with a $3 \mathrm{~Hz}$ artificial mode, which is equivalent to a ZV shaper. The rise time of the system equals the shaper duration, $\Delta_{1}=0.5 \mathrm{~s}$. Fig. 4 (b) shows the step command shaped by a command shaper designed with a $4 \mathrm{~Hz}$ artificial mode. In this case, the second impulse has a small negative value. The duration, $\Delta_{2}$, of the shaper is $0.4 \mathrm{~s}, 20 \%$ shorter than the $\mathrm{ZV}$ shaper in Fig. 4(a). Fig. 4(c) shows the step command shaped by a command shaper with a $5 \mathrm{~Hz}$ artificial mode. The first impulse is greater than 1 but the shaper duration is $33.3 \%$ less than the ZV shaper duration. Fig. 4(d) shows an even shorter duration shaper, whose artificial mode frequency and shaper duration, $\Delta_{4}$, are $6 \mathrm{~Hz}$ and $0.286 \mathrm{~s}$, respectively. In this case, all the magnitudes of impulses are greater than 1 .

Fig. 5 shows the simulated step responses subjected to the proposed command shapers with the artificial mode frequency varied. Increasing the artificial mode frequency clearly shortens the rise time.

Fig. 6 shows the sensitivity curves for the proposed command shapers, where $f_{t}^{d}$ represents the "true" damped natural frequency while $f_{s}^{d}$ is the damped natural frequency at which the proposed command shaper is designed. The figure indicates the range of frequencies over which the command shaper designed at one frequency adequately suppresses the vibration. This frequency suppression range is 
called the "insensitivity."

The figure shows that the $5 \%$ insensitivity gets a bit worse as the artificial mode frequency increases: e.g., from $0.076 \mathrm{~Hz}$ for $3 \mathrm{~Hz}$ to $0.055 \mathrm{~Hz}$ for $10 \mathrm{~Hz}$, a $27.63 \%$ decrease. However, it is apparent that the proposed shaper is still beneficial due to the advantage in rise time reduction. The shaper duration changes from $0.5 \mathrm{~s}$ for $3 \mathrm{~Hz}$ to $0.182 \mathrm{~s}$ for $10 \mathrm{~Hz}$. This gives a $63.7 \%$ decrease in shaper duration. Therefore, the proposed command shaper does not significantly degrade the robustness of command shaping, yet it shortens the rise time of the system greatly.

\section{ACKNOWLEDGMENT}

This work was financially supported by the Research Fund of Kumoh National Institute of Technology

\section{REFERENCES}

[1] O. J. M. Smith, Feedback Control Systems, McGraw-Hill Co., Inc., New York 1958.

[2] N. C. Singer and W. P. Seering, "Pre-shaping command inputs to reduce system vibration," ASME Journal of Dynamic Systems, Measurement, and Control, vol. 116, pp. 76-82, 1990.

[3] S. Lim, H. D. Stevens, and J. P. How, "Input shaping design for multi-input flexible systems," ASME Journal of Dynamic Systems, Measurement, and Control, vol. 121, pp. 443-447, 1999.

[4] W. E. Singhose and W. P. Seering, Command Generation for Dynamic Systems, Lulu.com, USA 2007.

[5] S. W. Hong, S. W. Park, and W. E. Singhose, "A method of input shaper design for residual vibration reduction using virtual mode concept," in Proceedings of the $15^{\text {th }}$ International Congress on Sound and Vibration, Daejon, Korea, 2008, pp. 1712-1718.

[6] S. W. Hong, J. Danielson and S. W. Park, "A new method for manufacturing machine vibration reduction using multi-mode input shapers," in Proceedings of the International Symposium on Flexible Automation, Atlanta, USA, 2008, no. 2008U-131.

[7] L. Y. Pao and W. E. Singhose, "Robust minimum time control of flexible structures," Automatica, vol. 34, pp. 229-236, 1998.

[8] T. Singh and G. R. Heppler, "Shaped input control of a system with multiple modes," ASME Journal of Dynamic Systems, Measurement, and Control, vol. 115, pp. 341-347, 1993.

[9] W. Singhose, N. Singer, and W. Seering, "Time-optimal negativeiInput shapers," ASME Journal of Dynamic Systems, Measurement, and Control, vol. 119, pp. 198-205, 1997.

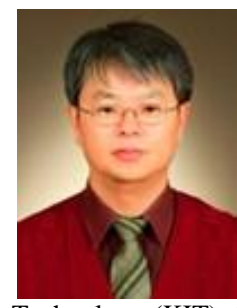

Seong-Wook Hong was born in Seoul, Korea in 1961. He received his B. S. degree in mechanical design and production engineering from Seoul National University, Seoul, Korea in 1983 and a M.S. degree and a Ph. D in mechanical engineering from KAIST, Daejon, Korea in 1985 and 1989 , respectively. He has worked as a senior researcher for Samsung Electro-Mechanics Co., Ltd., from 1989 to 1991. He joined Kumoh National Institute of Technology (KIT), 1 Yangho-dong, Gumi, Gyeongbuk 730-701, Korea, in 1991. He is currently a professor in mechatronics department at KIT. Prof. Hong published 89 peer-reviewed journal articles regarding rotor dynamics, structural vibration analysis and experiment, structural parameter identification, vibration control, and precision measurement for machine tools. His current research interest includes command shaping for positioning systems, spindle dynamics, vibration control, structural vibration analysis for mechanical systems and identification of parameters for machine tools.

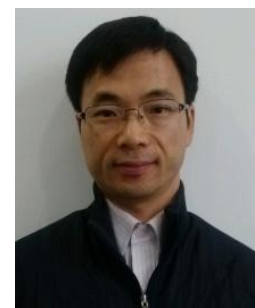

Yong-Kyu Seo was born in Chungnam, Korea, 1972. He received his B.S. and M.S. degrees in mechatronics from Kumoh National Institute of Technology (KIT) in 1995, 1997, respectively. He received his $\mathrm{Ph} . \mathrm{D}$ in mechatronics from KIT in 2009. Dr. Seo joined Top Engineering Co., Ltd., Paju, Gyeonggi 413-813, Korea, in 1997 and is currently a principal researcher to pursue development of LCD manufacturing machines.

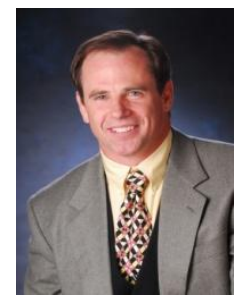

William Singhose received a Ph.D. from the Massachusetts Institute of Technology in June 1997. He then joined the faculty of the Woodruff School of Mechanical Engineering at the Georgia Institute of Technology. Dr. Singhose worked in industry for two and a half years before getting his Ph.D. He developed and installed control systems on industrial machines such as silicon-handling robots, coordinate measuring machines, and high precision air bearing positioning stages. While at Georgia Tech, Dr. Singhose has developed a worldwide network of research collaborations in the area of command shaping. The goal of this research community is to make computer-controlled machines move faster and more accurately by intelligently constructing the desired machine motion. Dr. Singhose has held visiting appointments at the Tokyo Institute of Technology, the Polytechnic University of Madrid, and MIT. 